\title{
Research on Applications of Wavelet Transform in Signal De-nosing
}

\author{
Haoming $\mathrm{Li}^{1, \text { a }}$ \\ ${ }^{1}$ Beijing University of Posts and Telecommunications, Beijing, China, 100876 \\ ahm@bupt.edu.cn
}

Keywords:Wavelet Transform; Signal De-nosing; Threshold

\begin{abstract}
The Wavelet transform is a new-style mathematic analysis tool, which is considered as a great breakthrough of tools andmethods recently. This paper introduces the theoretical basis of wavelet transform, clarifies methods of signal de-nosing based on wavelet transform and gives the characteristics of all the signal de-nosing methods to help the researchers to select.
\end{abstract}

\section{Introduction}

Proposal of Wavelet Transform. Wavelet transform is firstly proposed by a petroleum engineer named J.Morlet in 1974 in France. He established the inversion formula, but failed to get mathematicians recognized by physical intuition and signal processing the actual needs of the experience. Just as the 1807 French Thermal Engineer Fourier proposed that any function can be expanded into the infinite series of trigonometric functions and the concept of innovation has not been recognized. Belgian female mathematician Daubechies wrote the "Ten Lectures on Wavelets", whichhas played an important role in promoting the popularization of wavelet. Wavelet transform has a good time-frequency localization characteristics, which can effectively extract information from the signal. Therefore, the wavelet transform is known as the "mathematical microscope". It is a milestone in the development history of harmonic analysis.

Continuous Wavelet Transform.The wavelet transform is in limited time range changes and the average value for zero function. It is used to indicate the wavelet function, so it is called as wavelet function, which is recorded as $\psi(\mathrm{t})$.The function has a finite duration and the frequency and amplitude of the mutation, and its mean value is zero in a finite time range. In real life, people always study $F$ signal of finite energy.It is assumed that the function $f(t)$ and $L^{2}(R)$. Similarly, the wavelet function $\psi(\mathrm{t})$ and $\mathrm{L}^{2}(\mathrm{R})$. And Fourier transform or other integral transformation, only the existence of inverse transform in the practical application of meaning, but in general, the inverse transform does not necessarily exist. Wavelet analysis theory since the development, widely used in signal de-noising, image retrieval and pattern recognition and other fields, the requirements for wavelet transform can better reconstruct the original signal. The wavelet function $\psi(\mathrm{t})$ should satisfy the admissibility condition:

$$
C_{\psi}=\int_{0}^{+\infty} \frac{|\hat{\psi}(\omega)|^{2}}{\omega} d \omega(1)
$$

We call the wavelet function $\psi(\mathrm{t})$ as the mother wavelet.

Among them, the wavelet basis functions with two parameters of scale a and translation b.The function $\mathrm{f}(\mathrm{t})$ in the wavelet is based on the expansion, which is a time function projected onto the time scale plane. The scale corresponds to the frequency, the high frequency and the smaller scale. The time scale analysis method is more advantageous than the time - frequency analysis method to 
analyze the signal with singular point. The essence of wavelet transform is to filter the function $\mathrm{f}(\mathrm{t})$ and an active band pass filter. The width information of different time and frequency domain can be obtained under the wavelet transform, so as to realize the localization analysis of $\mathrm{f}(\mathrm{t})$. We can learn that after the wavelet transform coefficients with large amount of redundancy, it can use this redundancy to achieve signal de-noising and data recoveryfrom the continuous wavelet transform of the realization. Its dual wavelet is:

$$
\hat{\psi}(\omega)=\frac{\overline{\hat{\psi}}(\omega)}{\sum_{j=-\infty}^{\infty}\left|\widehat{\psi}\left(2^{-j} \omega\right)\right|^{2}}(2)
$$

Discrete Wavelet Transform. The concept of redundancy is usually used to measure whether the function family is orthogonal, if the signal loss part can transmit the same information amount, then the signal is redundant, the degree of redundancy is called redundancy. The scale factor a and the shift factor $b$ of the continuous wavelet transform are both continuous and redundant, so that the scale factor a and the shift factor $b$ can be discretized in order to reduce the redundancy. Now the problem is how to be discretized to get a spatial $\mathrm{L}^{2}(\mathrm{R})$ orthogonal wavelet basis. By the continuous wavelet transform time-frequency analysis that the quality factor of the wavelet invariant, so we can scale factor a in binary way discretization, obtained the dyadic wavelet and dyadic wavelet transform. Then, the time parameter b way binary integer times of discretization to get orthogonal wavelet function and the expression of wavelet series, and truly realize the wavelet transform of the continuous and discrete forms in the general functions of the complete reunification of the. For any integer $\mathrm{j}$, we record the dyadic wavelet function as:

$$
\psi_{\left(2^{-j}, b\right)}=2^{\frac{j}{2}} \psi\left(2^{j}(x-b)\right)(3)
$$

\section{Methods of Signal De-nosing Based on Wavelet Transform}

Donoho Threshold Method.Noise has the following characteristicsin the wavelet decomposition: if the signal $n(t)$ is a stable, zero mean white noise, the wavelet decomposition coefficients is not related to; if the signal $n(t)$ is a Gaussian white noise, the wavelet decomposition coefficients are independent, is also a Gaussian distribution; if the signal $\mathrm{n}(\mathrm{t})$ is a colored, smooth, zero mean Gaussian noise sequence, the wavelet decomposition coefficients is also a Gaussian sequence. For each scale, its coefficient is also a colored, stationary sequence. Donoho's waveletthreshold to noise method is the most widely used in engineering method. The basic idea is: a containing noise in the signal model can be expressed in terms of $F(t)=s(t)+n(t)$, in which $s(t)$ isthe original signal, $n(t)$ is theGaussian noise obeying $N\left(0, \sigma^{2}\right)$. Due to the wavelettransform is a linear transformation, the $\mathrm{f}(\mathrm{t})$ for discrete wavelettransform after the wavelet coefficient is still composed of two parts, one part is the signal corresponding to the wavelet coefficient, and the other part is the noise corresponding to the wavelet coefficient. Generally speaking, after wavelet decomposition, signal coefficient is higher than the noise coefficient, so you can find a suitable number of lambda as a threshold, when the decomposition coefficient is less than the critical threshold, that when the decomposition coefficients is mainly caused by noise, shall be abandoned; when the decomposition coefficient is greater than the critical threshold, that when the decomposition coefficients is mainly caused by the signal, put this part directly retained or according to a certain fixed amount to zero shrinkage, and then use the wavelet coefficients of wavelet reconstruction, is de noised signal. 
Translation Invariant Method.Translation invariant wavelet de-nosing method is the improvement of Donoho's threshold method, this method cannot only effectively suppress the pseudo Gibbs phenomena, and can reduce the original signal and estimate the signal between the root mean square errors to improve the signal-to-noise ratio. This method is used to deal with the fault signal of the gearbox, and the result is compared with the threshold method. The results show that the method can effectively remove the interference of the strong noise and extract the fault characteristic information of the gear, which has good engineering practicability. After the worst due to signal level random pulse of positive and negative pulse, and its size and signal discontinuity point position are closely linked, because a signal may contain several consecutive points, they will interfere with each other, but also for a discontinuous point of the optimal translation may be another discontinuous point of the translation. Therefore, we usually use the $\mathrm{n}$ times cycles translation instead of single time translation. The translation of signal with the threshold method for de-nosing processing, and then for each translation de-nosing results are averaged, namely so-called "translation de-nosing average" translation invariant wavelet de-nosing method. The method is able to show a better visual effect. It also can get a smaller root mean square error than the threshold method. At the same time, the signal noise ratio is improved.

Neighborhood Averaging Method.The neighborhood average method is to replace the gray level of each pixel with the average value of a few pixels. Effective suppression of additive noise, but it is easy to cause image blur, can be improved, mainly to avoid the smooth processing of the edge of the scene. It is a kind of local spatial domain processing algorithm. The processed image is:

$$
g(x, y)=\frac{1}{M} \sum_{(i, j) \in S} f(i, j)(4)
$$

$\mathrm{S}$ is a setof point $(\mathrm{x}, \mathrm{y})$ as the center of the neighborhood. $\mathrm{M}$ is the number of $\mathrm{S}$ coordinates. Image is processed by the neighborhood average method, which is relatively fuzzy, because the average is in exchange for the reduction of noise with the fuzzy of the image. The processing effect of the image neighborhood average method is related to the neighborhood radius. The larger the radius is, the greater the degree of fuzziness of the image. We image neighborhood average algorithm is simple and fast, but it can reduce the noise while making the image fuzzy, especially in the edge and details of the fuzzy very powerful. In it in the actual operation of the process does not need the statistical characteristics of image, so more convenient, the method of pulse jamming and salt and pepper noise have good inhibitory effect, and on the edge of the image can better protect.

Modulus Maximum De-noising Method.The first derivative of the smoothing function is used as the wavelet transform, and the modulus maxima of the wavelet transform at different scales correspond to the position of the signal mutation point. The smaller the scale of wavelet analysis, the smooth region of the smooth function is small, the corresponding value of wavelet coefficient modulus maxima point and the location of the mutation point is more accurate. However, wavelet transform has a very large impact on the small scale, which produces many pseudo extreme points, which cannot locate the location of the mutation point only by one scale. On the contrary, the noise is smoothed in large scale, and the extreme points are relatively stable, but because of the smoothing effect, the location of the noise is also produced. At the same time, only in the appropriate scale of each mutation point caused by wavelet transforms to avoid overlapping interference. Therefore, when the wavelet transform modulus maxima method is used to judge the abrupt point of the signal, it is needed to combine the multiple scales. The wavelet transforms modulus maxima are used to characterize the properties of the signal mutation at the multi scale. In many cases, the wavelet transform does not require all of the continuous scalein order to achieve a 
fast algorithm, the choice of scale by binary change, that is, binary conversion. The mutation point of the signal can generate the corresponding modulus maxima at different scales. The wavelet theory shows that the amplitude of the modulus maxima is determined by the local exponent of the signal at the point of the mutation.

\section{Selection of Methods of Signal De-nosingBased on Wavelet Transform}

Donohothreshold method is mainly applied to the signal in the signal mixed with white noise. The advantage of de-nosing by threshold method is that the noise is almost completely suppressed, and the characteristic peak of the original signal is well preserved. We use soft threshold de-nosing method can make the estimation of signal to achieve maximum to minimize the mean square error, that is, to noise estimation of signal is approximated optimal estimation of the original signal; and estimation of signal at least and the original signal also smooth and does not produce additional oscillation. Translation invariant wavelet de-nosing is mainly applied to the signal mixed with white noise and contains a number of points of discontinuity, is based on the threshold method of improvement. The disadvantage is that the computation speed is not fast.Neighborhood averaging algorithm is simple and fast calculation speed, but neighborhood averaging method is to blur the image in exchange for the cost of noise reduction, noise reduction and image blurring.Modulus maxima image de-nosing method is mainly suitable for the image mixed with white noise and image contains more singular points, can effectively retain the signal singularity information, the signal to noise without excess oscillation. Modulus maxima de-nosing method, wavelet transform coefficients only mold maximum value point, and the rest are set to zero. The reconstructed imageonly through the finite modelwill also have a large error. Although the modulus maximum de-noising method can eliminate the noiseaccurately, thecomplexcomputation will lead to slow speed.

\section{Conclusion}

The de-nosing method can effectively remove the random noise mixed in the one-dimensional stationary signal and two- dimensional image signal. The wavelet packet transform can not only decompose the low frequency part, but also decompose the high frequency part in the analysis of the high frequency. In this paper, the focus is the wavelet transforms de-nosing methods' introduction and selection. The research on wavelet packet de-noising is not deep in this paper. The wavelet packet transform de-noising is the direction of further research.

\section{References}

[1] Wang Fang, JiZhong, PengChenglin, Chinese Journal of Scientific Instrument, Vol. 34 (2013) No 5, p.1160-1166

[2] Chen Zhifu, Deng Juzhi, Chen Hui, Ni Peng, Chinese Journal of Engineering Geophysics, Vol. 9 (2012) No 6, p.732-737

[3] QinYahui, FengJinghui, ChenLiding, Information Technology, Vol. 17 (2010) No 1, p.732-737

[4] Chen Xiuzhi, Journal of Heilongjiang University of Science \& Technology, Vol. 24 (2014) No 6 p.638-641

作者: 李浩铭 
单位: 北京邮电大学

邮寄地址: 北京市海淀区西土城路 10 号北京邮电大学 李浩铭（收）

邮编: 100876

电话: 15510468786 